\title{
Improving the design of the earthquake-proof suspension building
}

\author{
Taliat Azizov ${ }^{1}$, Nadzieja Jurkowska ${ }^{2}$ \\ ${ }^{1}$ Pavlo Tychyna Uman State Pedagogical University, Uman, Ukraine \\ ${ }^{2}$ Tadeusz Kosciuszko Cracow University of Technology, Krakow, Poland
}

\begin{abstract}
The proposal for improving the constructive scheme of the supporting frame of the suspension building has been considered. The strains in its bearing elements due to earthquakes are significantly less than in the traditional cantilever buildings. In former schemes the vertical components of strains develop during horizontal oscillations of the grounds. This disadvantage has been eliminated now. It is shown that in the improved design of the structure under the earthquake the vertical component of vibrations is practically absent, due to arrangement of the suspension device strictly vertically. The numerical data for calculation results with variation of some characteristics of the supporting frame and suspensions have been shown in order to allow adjusting the dynamic strains at the vibrations of the base. The scheme for installation the vertical elements of the frame have been proposed. Its usage will allow to build the high-rise suspension buildings.
\end{abstract}

Keywords: suspension building, seismic safety, dynamic forces, Lagrange's equations of the second kind

\section{The problem statement}

The issue of protecting buildings subjected to earthquakes is always one of the most important tasks for design and construction in the seismic areas. The usage of the damping devices, disconnecting constraints is an effective protection measures [1-5]. However the dynamic forces from the action of the earthquake still large enough, so that strengthening of the building structures is required, and the cost of construction is increasing.

Another highly effective way for the substantial reduction of the dynamic forces acting on the bearing structures is the suspension of the building to the bearing frame $[6,7]$. According to the preliminary calculations by the simplified dynamic schemes, the suspension of the very building allows to reduce the loading of the bearing structures several times. The unusual solution to the problem of the suspension building entails facilitation in installation of structure of the bearing frame, improving its shape to reduce the vertical components of force during oscillation, and a study of change of the efforts to the support frame depending on various factors, including the length of the suspension thread and the stiffness of the supporting frame. 
Thus, the purpose of this article is to improve the structure of the supporting frame of a suspension building. The tasks presuppose development of a technique for its simple installation without usage of special tools and the analysis of the influence of some construction parameters on dynamic efforts.

\section{Improving the supporting frame of the suspension building}

The building proposed in [6] is a structure suspended on the bearing frame, which mass is much less than the mass of the very building. The preliminary calculations are conducted by the building mechanics' techniques using the Lagrange equations of the second kind. Herewith two options for the suspension of the building have been proposed: the pyramidal and the rectangular frames are used as the bearing structures (Figure 1). In both cases, the building itself is suspended to the support frame on the cables.
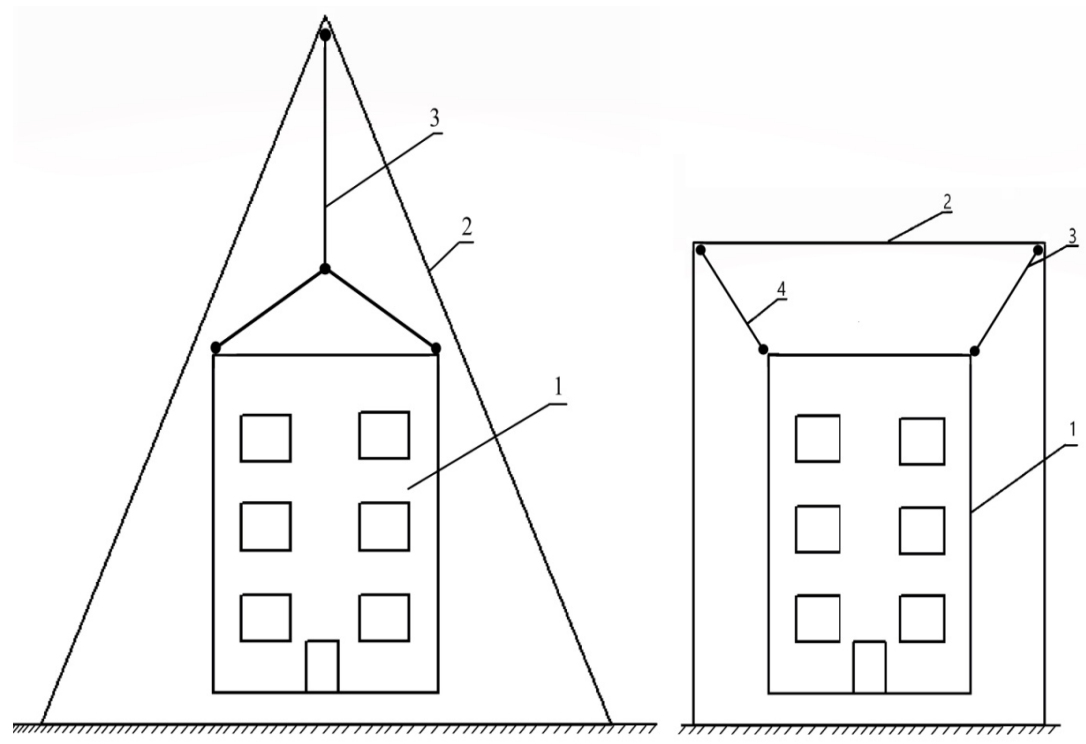

Fig. 1. The scheme of the suspension building:

a) suspension on the pyramid frame; b) suspension on the rectangular frame 1 - suspension building; 2 - frame; 3, 4- suspension (cables)

The advantage of the rectangular frame is that its area is much less than the area of the pyramidal frame. However the large vertical components of the strains develop in the cables during the horizontal oscillation, due to the inclined arrangement of the cables, on which the building is suspended.

The principle of the preliminary dynamic calculation for such a suspension building has been explained in [7]. Its purpose is to determine the equations of motion of the system and of the order of the seismic forces acting on the bearing frame. In this case, the whole suspension building (or structure) is considered as a point mass, suspended on the bearing frame. A simplified, but the most approximate dynamic calculation scheme for this case is presented in Figure 2. 


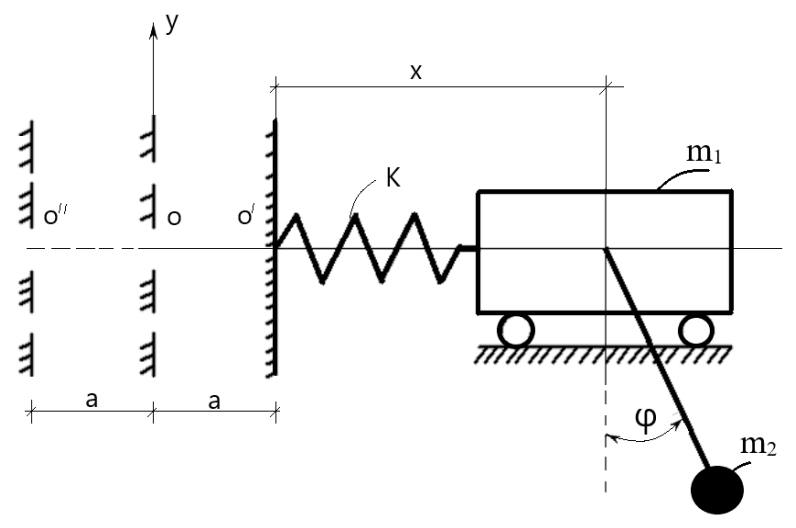

Fig. 2. Dynamic scheme with the oscillating support

The support located at the beginning of the coordinate system OXY, oscillates between the points $\mathrm{O}^{\mathrm{I}}$ and $\mathrm{O}^{\mathrm{II}}$ relatively to the equilibrium position $\mathrm{O}$ by the law $x=A \sin (p t)$, where $a$ is the amplitude, $p$ is the frequency. Amplitude and frequency of oscillations can be determined by seismograms, depending on the seismic zone.

The cart is attached to a support by a spring of stiffness $\mathrm{k}$. The conditional stiffness of the spring is determined from the condition $x_{c}=x_{s}$ (Figure 3).

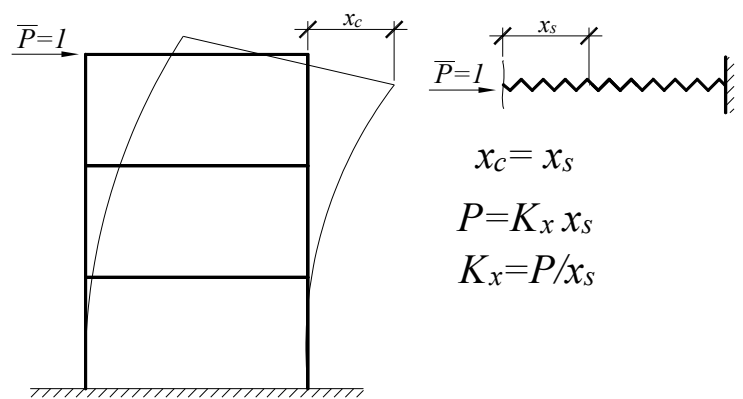

Fig. 3. Dynamic scheme with the oscillating support

The spring stiffness simulates the displacement of the free end of the frame in horizontal direction and is chosen from the condition of equality of the static deflection of the end of the cantilever (supporting frame) from a single horizontal force to the displacement of the spring from the same force. The base of the system (point $O$ in Figure 2) oscillates horizontally left and right with an amplitude $a=O-O^{I}$.

It should be noted that this scheme is valid for the pyramidal frame (construction according to Figure 1, a), but is not quite applicable for the design of the rectangular frame (Figure 1, b). The reason is that when using the scheme according to Figure 1, b, at the horizontal oscillation of the foundation considerable vertical stress components develop in the suspensions. They should be considered at the design. At the same time the pyramidal frame takes much place.

In order to eliminate this disadvantage, the structure of the bearing frame is proposed to be changed (Figure 4). The difference from the Figure 1,b is in the vertical placement of the suspensions 3 and 4, and in the arrangement of the additional bracing in the bearing frame. Therefore, during the horizontal oscillations the foundation will also oscillate in the 
horizontal direction, and the vertical component of dynamic stress will be practically absent.

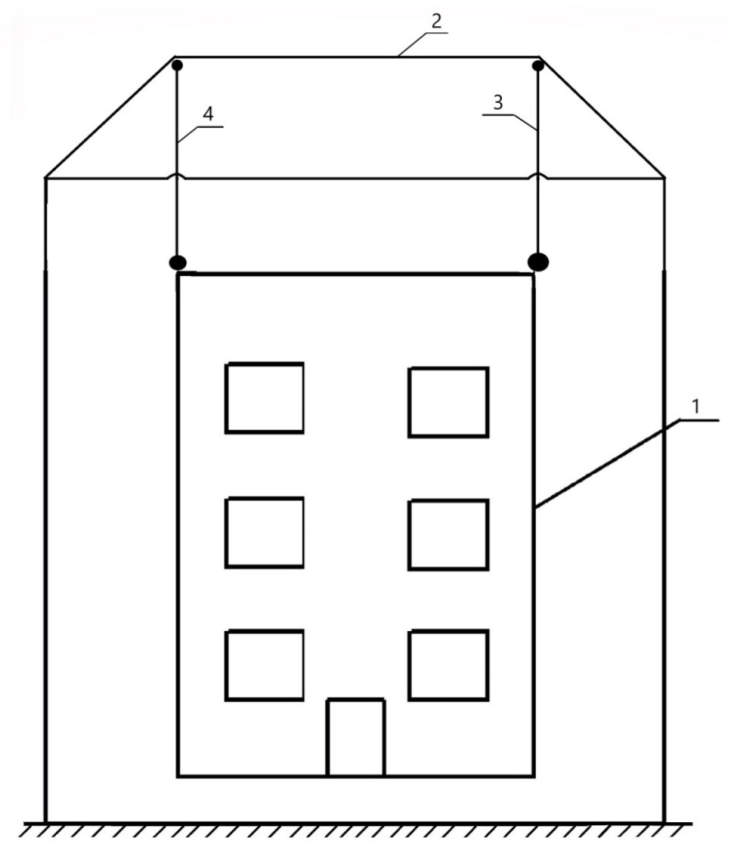

Fig. 4. The improved scheme of the bearing frame of the suspended building 1 - suspended building; 2 - the bearing frame; 3,4 - suspension (cables)

Since according to the scheme on the Figure 4 for the small oscillations of the foundation the vertical stresses will be absent in the suspensions of the system, the usage of the dynamic scheme represented on the Figure 2 will be effective.

This scheme has been calculated using the Lagrange equations of the second kind [7]. The derived system of equations is:

$$
\left\{\begin{array}{l}
\left(m_{1}+m_{2}\right) \ddot{x}+m_{2} l \ddot{\varphi} \cos \varphi-\left(m_{1}+m_{2}\right) p^{2} a \sin p t+k x=0 \\
\ddot{x} \cos \varphi+l \ddot{\varphi}-a p^{2} \sin p t \cos \varphi+g \sin \varphi=0
\end{array}\right.
$$

where, according to the Figure 2: $m_{1}, m_{2}$ are the masses of the trolley (simulating the bearing frame) and the suspension of the building, respectively; $\mathrm{x}$ and $\varphi$ are the generalized coordinates; $a$ and $p$ are the amplitude and frequency of oscillation of the base, respectively; $g$ is the acceleration of gravity.

The system of differential Lagrange's equations can be easily solved both numerically and analytically. Knowledge of the law of motion of this system allows to obtain the forces acting on the system's elements, including the spring (the horizontal component to the supporting frame in this case).

\section{The technique for installation the construction of the supporting frame}

The length of the columns of the bearing frame when suspending the high building is large enough. The techniques for installation the high-rise buildings by the increasing of separate sections or rising the building as a whole are well known. During the lifting entirely, the falling masts are used. Their height is accepted equal to about a third of the 
height of mounted support. In this regard the complexity of works and their cost increase. In case of installation of the supporting frame of the suspension building, it is possible to avoid the falling mast, simplifying the installation essentially. The arrangement scheme is shown in Figure 5.

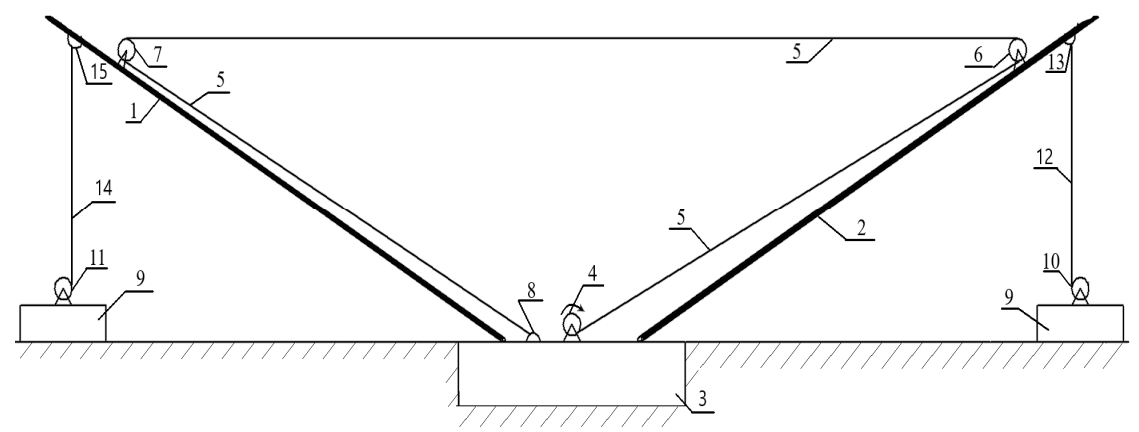

Fig. 5. The arrangement of installation of the columns of the supporting frame of the suspension building

The pillars left 1 and right 2 of the paired columns of the bearing frame are pivotally mounted to the monolithic foundation 3 (in the direction perpendicular to the drawing, the columns are coupled, i.e. two columns rise right, and two columns rise left). The free ends of the columns 1 and 2 should be lifted beforehand to the heights $h$ and placed on temporary supports. The winch 4 is installed on the foundation. The cable of the winch 5 is held through the block 6 fixed on the right column, and through the block 7 fixed on the left column, and is attached to the foundation at the point 8 . Thus, it is possible to lift simultaneously both of the coupled columns.

In order to eliminate the uneven lifting, the temporary counterweights 9 are mounted under the columns. The winches 10 (at the right column) and 11 (at the left column) are fixed on these columns. The cable 12 from the winch 10 is mounted at the point 13 of the column on the right. Similarly, the cable 14 from the winch 11 is attached at point 15 on the left column.

When the leading winch 4 is enabled, in case the balance is disturbed and one of the columns rises more than the other, the balance should be recovered by means of wires 12 and 14. During the installation process the cables emerging from winches 10 and 11 should be tightened. Such a scheme allows to lift both of coupled columns simultaneously, as well as prevents rollover of the system.

The arrangement scheme (see Figure 5) is very effective. The presence of the unit 6 , through which the lifting cable 5 is mounted to the opposite column, sharply reduces the stress required for lifting of both columns. It can be easily seen from the design diagram, presented in Figure 6 (the right column is presented, the left column will be symmetrical). 


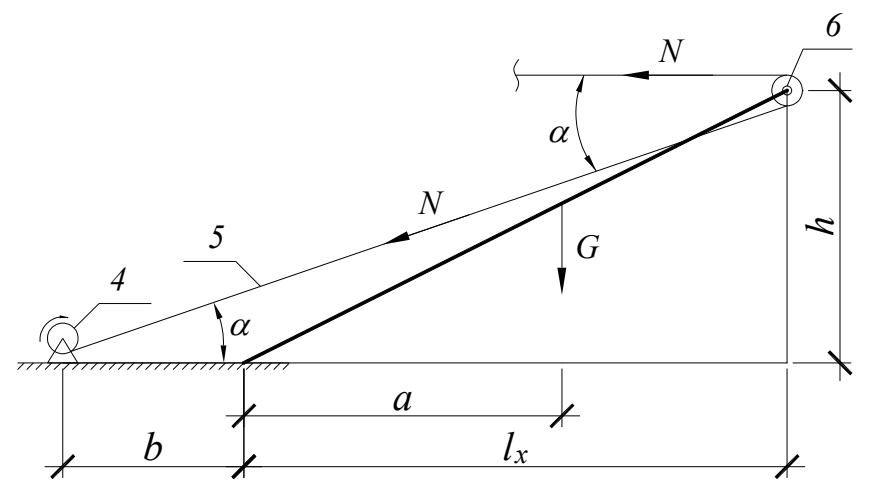

Fig. 6. The design scheme for lifting the columns to the design position

Considering the power winch (p.4 in Figure 6), the strains in the cables can be calculated from equilibrium conditions. There are two options: 1 . for the cable attached at point 6 of the right support and not thrown to the left support (see also Figure 5); 2. by the proposed scheme using units 6 and 7 , and with the cable attached at point 8 .

In order to fix the elevating part of each column in vertical position, the cantilever elements must be welded to them. Those cantilever elements must be perpendicular to the elevating columns' parts and of the length equal to half the distance between columns. After bringing columns to the vertical position the cantilever elements of two columns are fastened together by bolts or welding.

The calculations using equation (1) demonstrate several times decreasing of the strains in the bearing structures of the suspension building, comparatively to the strains in the building constructed by the traditional cantilever scheme.

\section{The impact of individual factors on the stresses in the system}

The calculation has been conducted for the scheme of the suspended building represented in Figure 2, on the basis of solution for the derived system of Lagrange's equations of the second kind (1). Initial data: the mass of the trolley (i.e. the mass of the bearing frame) $\mathrm{m} 1=51000 \mathrm{~kg}$, mass of the building $\mathrm{m} 2=1612000 \mathrm{~kg}$. These data were obtained by a preliminary rough calculation of the five-storey building with dimensions $12 \times 12 \mathrm{~m}$ and a total mass $750 \mathrm{~kg} / \mathrm{m} 2$ (including the mass of structures, columns, floors, etc.). The columns of the supporting frame are accepted from steel hollow tubes with a thickness of $10 \mathrm{~mm}$. The basic value of the stiffness coefficient of the conditional spring $\mathrm{k}$ (the stiffness of the supporting frame in the horizontal direction) was determined from the calculation of the bearing frame on a horizontal unit load, and is equal to $\mathrm{kb}=2303000 \mathrm{~N} / \mathrm{m}$. Let the oscillation amplitude of the ground $\mathrm{a}=0.048 \mathrm{~m}$, the oscillation frequency $\mathrm{p}=10 \mathrm{~Hz}$; the maximum acceleration of the ground vibration $\mathrm{w}=4.8 \mathrm{~m} / \mathrm{s} 2$ maximum velocity of ground vibration $v \max =0.48 \mathrm{~m} / \mathrm{s}$ (approximate data for the 9 magnitude earthquake by the Richter scale).

For the suspension thread of length $5 \mathrm{~m}$ the change of the generalized coordinates $x=x(t)$ and $\varphi=\varphi(t)$ for the first five seconds of oscillations is shown in Figure 7. 

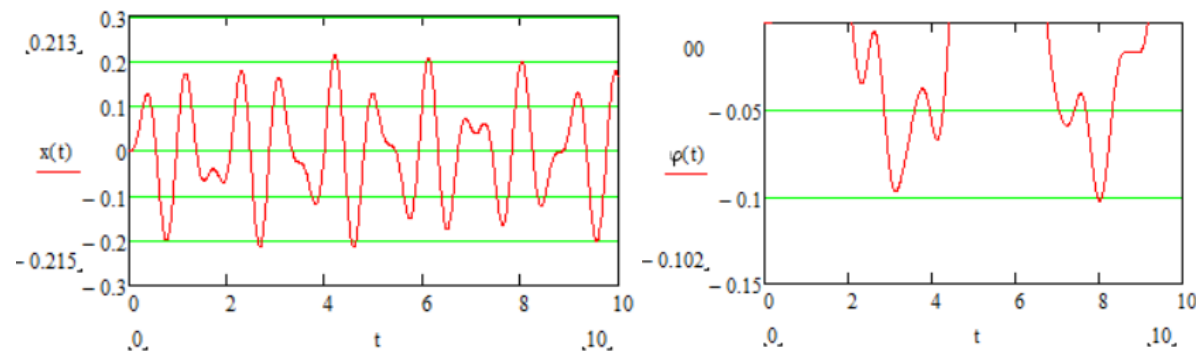

Fig. 7. Graphs of change

a) of the variable $x=x(t)$ of the displacement of the point of the frame's suspension

b) of the rotation angle $\varphi=\varphi(t)$ of the thread suspension

The rotation angle is sufficiently small, i.e. even in case of strong earthquake deviation of the buildings from the vertical position does not exceed $4^{\circ}$. Herewith the horizontal force is small enough.

From the solution of the system of differential equations (1), the oscillation dependences $x=x(t)$ of the conditional truck (Figure 2) have been obtained. The maximum values xmax are taken from these dependences. The maximum horizontal force on the bearing frame is $F_{\max }=x_{\max } \cdot k$. Meanwhile, for the cantilever (traditional) building with acceleration of oscillation of ground $\mathrm{w}=4.8 \mathrm{~m} / \mathrm{s} 2$ the total horizontal force will be equal to $F_{t r}=w \cdot m_{2}=7737600 \mathrm{~N}$.

The table 1 provides the calculated data for different length of the suspension of building $l$ (the length of the suspension cables) and different stiffness of the supporting frame $k$, which is accepted for both less and greater than the value basic $k_{b}$ (see above). The values of the ratio of maximum horizontal force of the traditional cantilever building $F_{t r}$ to the horizontal force of the suspension building $F_{\max }$ are presented in the last column.

Table 1. Ratio of maximum horizontal forces of the cantilever and suspension buildings.

\begin{tabular}{|c|c|c|c|c|c|c|}
\hline № & $l, \mathrm{~m}$ & $k, \mathrm{~N} / \mathrm{m}$ & $x_{\max }, \mathrm{m}$ & $F_{t r}, \mathrm{~N}$ & $F_{\max }, \mathrm{N}$ & $F_{t r} / F_{\max }$ \\
\hline 1 & 3 & $k=k_{b}=2303000$ & 0.235 & 7737600 & 541205 & 14.29 \\
\hline 2 & 5 & $k=k_{b}$ & 0.213 & 7737600 & 490539 & 15.77 \\
\hline 3 & 7 & $k=k_{b}$ & 0.213 & 7737600 & 490539 & 15.77 \\
\hline 4 & 10 & $k=k_{b}$ & 0.198 & 7737600 & 455994 & 16.97 \\
\hline 5 & 12 & $k=k_{b}$ & 0.201 & 7737600 & 462903 & 16.71 \\
\hline 6 & 15 & $k=k_{b}$ & 0.204 & 7737600 & 469812 & 16.46 \\
\hline 7 & 5 & $k=k_{b}{ }^{*} 0.6$ & 0.178 & 7737600 & 245960 & 31.45 \\
\hline 8 & 5 & $k=k_{b} * 0.7$ & 0.202 & 7737600 & 325644 & 23.76 \\
\hline 9 & 5 & $k=k_{b}{ }^{*} 0.8$ & 0.187 & 7737600 & 344529 & 22.46 \\
\hline 10 & 5 & $k=k_{b} * 0.9$ & 0.202 & 7737600 & 418685 & 18.48 \\
\hline 11 & 5 & $k=k_{b}{ }^{*} 1.1$ & 0.252 & 7737600 & 638391 & 12.12 \\
\hline 12 & 5 & $k=k_{b} * 1.2$ & 0.273 & 7737600 & 754463 & 10.25 \\
\hline 13 & 5 & $k=k_{b}{ }^{*} 1.3$ & 0.231 & 7737600 & 691591 & 11.19 \\
\hline 14 & 5 & $k=k_{b} * 1.4$ & 0.241 & 7737600 & 777032 & 9.95 \\
\hline 15 & 5 & $k=k_{b} * 1.5$ & 0.265 & 7737600 & 915442 & 8.45 \\
\hline
\end{tabular}

The table demonstrates the possibility of reducing the horizontal forces by reducing the stiffness of the bearing frame. Herewith the frequency and period of oscillation of the system also change.

In order to exclude resonance phenomena during an earthquake, the strains in the bearing frame, as well as the frequency and period of oscillation of such a building can be 
regulated by changing the length of thread of the suspension, stiffness of the bearing frame in the horizontal direction, 2 ratio of the mass of the frame and the period of oscillation of such a building.

Calculations illustrate that the horizontal component of the dynamic force on the bearing frame of the suspension building during horizontal oscillation of the ground is significantly less than the similar strength to the traditional cantilever building. Moreover, unlike the protection systems with disconnected constrains, the proposed system does not require replacement of any structural elements after the earthquake.

One of essential advantages of these proposals is the possibility to conduct numerous preliminary calculations by the approached schemes. Thus the most acceptable design option for the structure of the bearing frame, suspensions and other parameters can be evaluated. After the preliminary determination of the structural scheme, the final calculation of strength, stability and deformability can be conducted using the known software systems such as Ansys, Abacus etc.

\section{Conclusions and perspectives of research}

The mass of the proposed suspension building is many times greater than the mass of the supporting frame. This factor together with the design features of the proposed kinematic scheme of the building allow to reduce dynamic forces in bearing structures of the building during an earthquake significantly. The article demonstrates that the change in stiffness of the supporting frame in the horizontal direction results in regulation of strains in the bearing structures, the frequency and period of oscillation of the building during an earthquake. Additionally, the proposed technique of installation of the supporting frame allows to construct buildings of such design, including high-rise buildings without the use of sophisticated equipment, helicopters, etc. In totality, this makes the proposed building attractive both from a perspective of seismic safety, and from the position of relative simplicity of construction.

In future it is planned to continue improvement of techniques of dynamic calculation of the suspension buildings, analysis of impact of various factors on the strains, deformability, the frequency of oscillation of the building during the earthquake, as well as the development of various details of the proposed structural system.

\section{References}

1 C.C. Chang, Mass dampers and their optimal designs for building vibration control, Eng. Struct. 21(5) (1999)

2 Y. Eisenberg, Constructions with disconnective constraints for seismic areas [Sooruzheniya s vyklyuchayushchimisya svyazyami dlya sejsmicheskih rajonov]. Moscow (1976).

3 R.O. Hamburger, Facts for Steel Buildings Number 3 - Earthquakes and Seismic Design, 71 (2009).

4 K.S. Moon Structural Design of Double Skin Facades as Damping Devices for Tall Buildings, Procedia Eng. (2011) 1351-1358.

5 Eurocode 8: Seismic Design of Buildings Worked examples, (2012).

6 T. Azizov, Design of the earthquake-resistant buildings [Konstrukciya sejsmichno stijkoï budivli]. Patent for the useful model Nr 54247. Ukraine, Bulletin Nr 20.

7 T. Azizov, Dynamic design scheme of suspended seismically safe buildings, in: Sci. Eur. 17, Vol 1 (2017) 